\title{
Editorial: Hormones and Life History Strategies
}

\author{
Sarah McMenamin ${ }^{1 *}$, Fedor Shkil ${ }^{2}$ and Takashi Koyama ${ }^{3}$ \\ ${ }^{1}$ Biology Department, Boston College, Chestnut Hill, MA, United States, ${ }^{2}$ AN Severtsov Institute of Ecology and Evolution, \\ RAS, Moscow, Russia, ${ }^{3}$ Section for Cell and Neurobiology, Department of Biology, University of Copenhagen, Copenhagen, \\ Denmark
}

Keywords: hormone, reproduction, metamorphosis, endocrine factors, thyroid hormone, serotonin, life history

\section{Editorial on the Research Topic}

Hormones and Life History Strategies

\section{INTRODUCTION}

Considering the hormonal contributions to life history strategies opens an exciting area of integrative research within evolutionary ecology. Through the course of its life, an organism must appropriately allocate resources between survival, growth, maturation and reproduction: these patterns are captured in the animal's life history. Life history encompasses timing of reproduction and number of offspring, and these characteristics ultimately determine a population's size and structure, shaping the ecology, and evolution of a species. Hormones are systemic signaling molecules that coordinate cellular activities between disparate organ systems. Endocrine factors mediate life history tradeoffs and transitions across diverse metazoan species. Indeed, hormones regulate the timing of hatching or birth, determine the tempo of development, trigger the onset of ecological transformations such as metamorphosis, and regulate reproductive cycles (see Figure 1). By coupling hormonal axes to the environment-including stimuli from social, habitat or resource conditions-many species have evolved plastic life history transitions that can adaptively adjust to match external conditions. Thus, research which integrates both endocrinology and life history holds considerable promise at the frontier of evolution and ecology.

In this special issue, researchers explore hormonal contributions to life history transitions and traits at different developmental stages (Figure 1; each citation is placed outside the life cycle at a stage relevant to the work). Reviews and original research from this issue focus on diverse animals across the metazoan phylogeny, from mollusks to mammals. Researchers have integrated perspectives and techniques from multiple biological disciplines which will be of interest to ecologists, evolutionary and organismal biologists, and comparative endocrinologists. In this editorial, we highlight some of the key interpretations and findings of these manuscripts, identify some of the common themes that emerge, and emphasize the promise of future research in this area.

\section{HORMONAL REGULATION OF DIVERSE LIFE HISTORIES}

Received: 17 December 2021

Accepted: 19 January 2022

Published: 11 February 2022

Citation:

McMenamin S, Shkil F and Koyama T (2022) Editorial: Hormones and Life History Strategies.

Front. Ecol. Evol. 10:838555. doi: 10.3389/fevo.2022.838555

Despite having vastly different life cycles, invertebrates as diverse as sea urchins, fruit flies and freshwater mollusks have each evolved hormonal triggers to regulate life history transformations; many of these stimulating endocrine triggers couple external environmental conditions with genomic and developmental responses. Voronezhskaya reviews the roles of a neuroendocrine factor-specifically, maternally-provided serotonin-in shaping the developmental and phenotypic plasticity of freshwater gastropod mollusks under seasonal environmental changes. Environmental cues, including temperature and daylight length, influence life history in insects through altering 


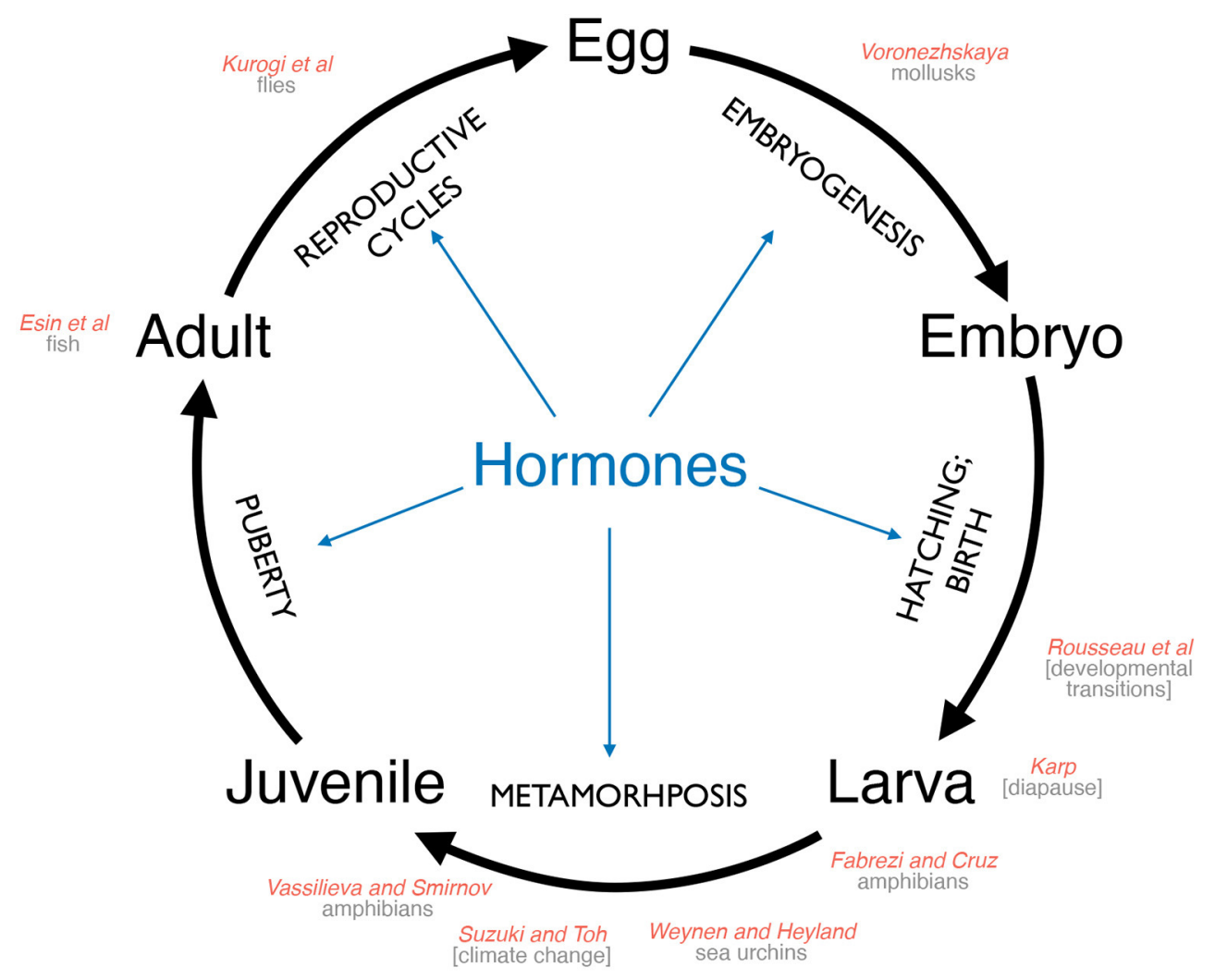

FIGURE 1 | A generalized metazoan life cycle. Transitions between each stage of life history require processes that involve hormones (shown in blue). The nine manuscripts in this special issue tackle different components of the metazoan life cycle in highly divergent organisms; each manuscript is cited (in red) near one of the life cycle stages or transitions on which the authors focus. The primary organism or broad subject of each manuscript is listed below in gray.

biosynthesis of juvenile hormone. Kurogi et al. review the roles of juvenile hormone as a mediator between environmental conditions and seasonal reproductive dormancy in $D$. melanogaster. Wynen and Heyland advance the idea that endocrine mechanisms regulate programmed cell death during the metamorphic transition of sea urchins, detailing the molecular basis of these processes and the relationships with environmental cues.

Since hormonal axes and the life history characteristics they control can be highly responsive to environmental conditions, changing climate should systematically (and catastrophically) alter life history patterns. Implications of global climate change on metamorphic organisms-both vertebrates and invertebrates-are explored in depth in this issue by Suzuki and Toh. These authors develop the concept of a "developmental goblet," which narrows not only at the phylotypic stage during embryogenesis, but again in post-embryonic development during the metamorphosis. The developmental and endocrine constraints of metamorphosis are predicted to be highly sensitive to changing ecological conditions, presenting both constraints, and opportunities for organismal adaptation (Suzuki and Toh).

Dormancy represents a discrete and dramatic type of plastic life history adaptation: under unfavorable environmental conditions, some organisms can enter a state of diapause, during which developmental, physiological, and reproductive functions are arrested. Karp surveys the endocrine mediators stimulating diapause across metazoan species, focusing specifically on nematodes, and certain specialized species of insects and fishes.

Original research in this issue tackles the question of how the endocrine factors influence life cycles and development in vertebrates. Many vertebrates-specifically amphibians and teleost fishes-undergo discrete metamorphic transitions stimulated by thyroid and corticosteroid hormonal axes. Moreover, egg hatching (in reptiles and birds) and birth (in mammals) represent profound developmental transitions that are similarly regulated by endocrine factors. Rousseau et al. compare and contrast these different types of vertebrate developmental transitions from a hormonal perspective. Esin et al. present evidence that thyroid hormone axis played a role in the ecological niche specialization and emergence of adaptive metabolic traits in three species of extremophile arctic salmonids. Fabrezi and Cruz experimentally altered thyroid production in an Argentinian frog, demonstrating that thyroid hormone modulates developmental rate and timing of metamorphosis in the species. Finally, Vassilieva and Smirnov ask whether amphibians show evolutionary trends in the hormonal controls of skeletal metamorphosis, highlighting the importance of endocrine factors in adaptation. 


\section{OUTLOOK}

Although life history strategies are phenomenally diverse across metazoans, life cycles can be generalized into certain common stages, and transitions between these stages are all regulated by different hormonal axes (see Figure 1). Indeed, hormones influence nearly every aspect of organismal physiology across the life cycle. The collection of reviews and original research presented in this special issue emphasize the fact that numerous endocrine cascades regulate a variety of life history transitions (birth, metamorphosis, diapause) in myriad animal species. Many important questions remain: Are hormone-mediated life history transitions evolutionarily homologous between diverse species? What are the molecular mechanisms underlying ecologically-cued reproductive cycles? What are the hormonal contributions to subtle developmental transformations? How are hormonal cues interpreted into genomic and cellular activities? Such questions will provide rich areas for future investigation. Like the work presented in this special issue, the strongest future efforts will utilize and integrate the strengths of traditional model organisms (Xenopus, Drosophila) with the diversity and broad relevance of diverse non-model species. Emerging technologies continue to accelerate integrative and holistic research, and we anticipate that these types of interdisciplinary approaches will be essential in advancing a full understanding of developmental and ecological transitions, and the responsiveness of these transitions to a changing climate and ecosystem.

\section{AUTHOR CONTRIBUTIONS}

SM wrote and edited the manuscript. FS and TK edited the manuscript. All authors contributed to the article and approved the submitted version.

\section{ACKNOWLEDGMENTS}

We thank Dr. Vincent Laudet, the members of each of our labs and all the researchers who submitted their work to this special issue topic. We are also grateful for the helpful comments of our Review Editor.

Conflict of Interest: The authors declare that the research was conducted in the absence of any commercial or financial relationships that could be construed as a potential conflict of interest.

Publisher's Note: All claims expressed in this article are solely those of the authors and do not necessarily represent those of their affiliated organizations, or those of the publisher, the editors and the reviewers. Any product that may be evaluated in this article, or claim that may be made by its manufacturer, is not guaranteed or endorsed by the publisher.

Copyright $\odot 2022$ McMenamin, Shkil and Koyama. This is an open-access article distributed under the terms of the Creative Commons Attribution License (CC BY). The use, distribution or reproduction in other forums is permitted, provided the original author(s) and the copyright owner(s) are credited and that the original publication in this journal is cited, in accordance with accepted academic practice. No use, distribution or reproduction is permitted which does not comply with these terms. 